\title{
Telessaúde em um centro de referência em Diabetes Mellitus: uma análise transversal
}

\author{
Telehealth in a reference center in Diabetes Mellitus: a cross-sectional analysis \\ Telesalud en un centro de referencia en Diabetes Mellitus: un análisis transversal
}

\author{
Ana Carolina Schroder ${ }^{1}$ (B) \\ Ana Paula Vanz ${ }^{2}$ (C) \\ César Geremia ${ }^{3}$ \\ Carolina Sturm Trindade ${ }^{1}$ (c) \\ Simone Travi Canabarro ${ }^{1}$ (c)
}

1. Universidade Federal de Ciências da Saúde de Porto Alegre. Porto Alegre, RS, Brasil.

2. Faculdades Integradas de Taquara. Taquara, RS, Brasil.

3. Hospital Nossa Senhora da Conceição. Porto Alegre, RS, Brasil.
Autor correspondente: Ana Carolina Schroder E-mail: anaschroder97@gmail.com

Recebido em 13/03/2020.

Aprovado em 07/07/2020.

DOI:https://doi.org/10.1590/2177-9465-EAN-2020-0046

\section{Resumo}

Objetivo: Analisar uma ferramenta de telessaúde de um centro de referência em Diabetes Mellitus sob a ótica dos cuidadores. Método: Estudo transversal, com abordagem quantitativa, desenvolvido em um centro de referência em Diabetes Mellitus, com cuidadores de crianças e adolescentes que utilizaram a Hot-Line e responderam ao questionário online, no período de novembro de 2018 a fevereiro de 2019. Resultados: A amostra foi constituída de 90 participantes, sendo 76 (84,4\%) do sexo feminino, tendo como o maior parentesco o materno 68 (75,6\%). Quanto aos motivos das ligações, $31(34,4 \%)$ referem-se às orientações gerais, 41 (45,6\%) ao ajuste de dose de insulina, 6 (6,7\%) à hipoglicemia aguda, 6 (6,7\%) à hiperglicemia aguda $2(2,2 \%)$ aos dias de doença e $4(4,4 \%)$ referem-se aos resultados de exames. Os participantes apresentaram um alto índice de satisfação com o uso da linha telefônica. Conclusão e implicações práticas: O teleatendimento produz benefícios imediatos aos pacientes, sendo resolutivo no manejo da doença. Cabe ressaltar que o uso da telessaúde como forma de promoção da saúde contribui para a prevenção de agravos de maneira rápida, satisfatória e sem o deslocamento do paciente e sua família.

Palavras-chave: Diabetes Mellitus; Telemedicina; Enfermagem; Criança; Adolescente.

\section{Abstract}

Objective: To analyze a telehealth tool of a reference center in Diabetes Mellitus from the caregivers' perspective. Method: A cross-sectional study, with a quantitative approach, developed at a reference center in Diabetes Mellitus, with caregivers of children and adolescents who used the Hot-Line and answered the online questionnaire, from November 2018 to February 2019. Results: The sample consisted of 90 participants, 76 (84.4\%) were female, and the highest kinship was the maternal 68 (75.6\%). Regarding the reasons for the telephone callings, 31 (34.4\%) refer to general guidelines, $41(45.6 \%)$ to the insulin dose adjustment, $6(6.7 \%)$ to the acute hypoglycemia, $6(6.7 \%)$ acute hyperglycemia, $2(2.2 \%)$ to the days of disease and $4(4.4 \%)$ refer to the test results. The participants presented a high level of satisfaction with the use of the telephone line. Conclusion and implications for practice: The call center produces immediate benefits to patients, being effective in the management of the disease. It should be emphasized that the use of telehealth as a form of health promotion contributes to the prevention of health problems in a fast, satisfactory way and without the displacement of the patient and his family.

Keywords: Diabetes Mellitus; Telemedicine; Nursing; Child; Adolescent.

\section{Resumen}

Objetivo: Analizar una herramienta de telesalud de un centro de referencia en Diabetes Mellitus desde la perspectiva de los cuidadores. Método: Estudio transversal, con un enfoque cuantitativo, desarrollado en un centro de referencia en Diabetes Mellitus, con cuidadores de niños y adolescentes que utilizaron la Hot-Line y respondieron al cuestionario en línea, de noviembre de 2018 a febrero de 2019. Resultados: La muestra estaba compuesta por 90 participantes, de los cuales $76(84,4 \%)$ eran mujeres y 68 $(75,6 \%)$ eran la madre. En cuanto a las razones de los enlaces, $31(34,4 \%)$ se refieren a las directrices generales, $41(45,6 \%)$ al ajuste de la dosis de insulina, $6(6.7 \%)$ a la hipoglucemia aguda, $6(6,7 \%)$ a la hiperglucemia aguda, $2(2,2 \%)$ a los días de enfermedad y $4(4,4 \%)$ se refieren a los resultados de las pruebas. Los participantes presentaron un alto nivel de satisfacción con el uso de la línea telefónica. Conclusión e implicaciones para la práctica: La teleasistencia produce beneficios inmediatos a los pacientes, y es resolutivo en el manejo de la enfermedad. Es importante destacar que el uso de la telesalud como forma de promoción de la salud contribuye a la prevención de agravios de manera rápida, satisfactoria y sin el desplazamiento de paciente y su familia.

Palabras clave: Diabetes Mellitus; Telemedicina; Enfermería; Niño; Adolescente. 


\section{INTRODUÇÃO}

O número de indivíduos que apresenta condições crônicas tem aumentado ao longo dos anos. Uma das mais importantes doenças crônicas é o Diabetes Mellitus (DM). O DM é considerado um grupo heterogêneo de distúrbios metabólicos que apresenta a hiperglicemia como sintoma em comum, como consequência de defeitos tanto na ação quanto na secreção da insulina no organismo. ${ }^{1}$ Por possuir alta prevalência e incidência em esfera mundial, o DM configura-se como uma epidemia. Estima-se que, mundialmente 451 milhões de pessoas possuam esta patologia e que, caso a tendência persista, 693 milhões apresentarão diabetes em 2045. ${ }^{2}$ No Brasil, as taxas do DM também são crescentes, com potencial de se transformar em um grave problema de saúde pública. Considerando as fases da infância e da adolescência, os dados sugerem que há, aproximadamente, 88.300 casos de crianças e adolescentes com Diabetes Mellitus do tipo 1 (DM1), contribuindo para que o País ocupe a terceira posição em ranking global sobre a incidência e prevalência na faixa etária abaixo de 20 anos. $^{2}$

O DM1 é a forma que mais afeta a infância, sendo caracterizado pela destruição total das células-beta no pâncreas, com origem autoimune. ${ }^{3}$ Com relação ao Diabetes Mellitus do tipo 2 (DM2), embora representando um quantitativo inferior, o número de crianças apresentando esta patologia vem-se tornando cada vez mais comum. Esta situação pode ser explicada devido aos níveis elevados de obesidade infantil, falta de atividades físicas e estilo de vida. ${ }^{4}$

Diante das alterações contínuas e incômodas para a criança, resultando em uma restrição na qualidade de vida e provocando um impacto direto no relacionamento e convívio com a família, eleva-se o grau de complexidade no atendimento pela equipe multiprofissional, necessitando, por sua vez, de diferentes meios de auxiliar na condição crônica. É ideal que o cuidado seja ampliado e que se encontrem novas maneiras e tecnologias para contemplar as necessidades e particularidades dos pacientes pediátricos durante o cotidiano, que recaem sobre a família. ${ }^{5}$

Frente a essa realidade, o reconhecimento e a valorização do uso de tecnologias em saúde vêm aumentando, uma vez que possibilitam ampliar e melhorar a assistência aos pacientes nos serviços de saúde. Nesse contexto, dentre as diversas modalidades de tecnologia disponíveis na telessaúde, encontram-se as linhas telefônicas, também denominadas Hot-Line, as quais permitem fornecer informações por meio de comunicação entre diferentes sujeitos, com o objetivo de monitorar a condição de saúde. ${ }^{6,7}$

As intervenções por meio de uma Hot-Line podem trazer inúmeras vantagens. Dentre elas, destacam-se a facilidade de acesso, não importando a distância geográfica entre o usuário e o serviço especializado, a redução significativa da utilização de serviços de emergências, a diminuição do tempo de permanência em internação, bem como o número de reinternações, uma vez que é possível aproximar a assistência ao paciente, com orientações focadas para a condição clínica no momento da ligação. ${ }^{8,9}$ Todos estes benefícios favorecem um controle maior, em se tratando de custos financeiros para o sistema de saúde como um todo, uma vez que os gastos em saúde para os pacientes diabéticos podem chegar a três vezes mais do que para aqueles sem diabetes..$^{10}$

Por se tratar de uma doença que acarreta alterações significativas para o paciente e sua família, a educação em saúde adquire uma importância fundamental no cotidiano das crianças e adolescentes como forma de auxiliar no autocuidado e enfrentamento de desafios para o controle adequado do DM. ${ }^{11}$

As práticas educativas, consideradas uma das formas de atuação por meio da utilização de linhas telefônicas, representam uma estratégia de aprendizagem, apoio e independência, contribuindo para uma redução de complicações e incentivo à adesão ao tratamento. Neste contexto, a participação da equipe multiprofissional proporciona um envolvimento e diferencial para o manejo adequado do diabetes, sendo imprescindível para a criação de vínculos e para estimular mudanças no estilo de vida, impactando diretamente o sucesso e desenvolvimento relacionados ao tratamento e à doença. ${ }^{12,13}$

Assim, o presente estudo tem por objetivo analisar uma ferramenta de telessaúde de um centro de referência em Diabetes Mellitus sob a ótica dos cuidadores.

\section{MÉTODO}

Trata-se de um estudo descritivo, transversal com abordagem quantitativa, realizado em um Centro de Referência em Diabetes Mellitus, localizado em uma capital no sul do país. O local é referência na América Latina em orientação preventiva de complicações, através de práticas de tratamento com oferta de novas tecnologias e educação em saúde para crianças e adolescentes com diabetes. Os pacientes são atendidos por uma equipe multiprofissional composta de endocrinologistas, nefrologistas, pediatras, oftalmologistas, ginecologistas, psiquiatras, enfermeiras, nutricionistas, psicólogas, odontólogos, assistentes sociais e educador físico.

A população participante do estudo foi constituída de cuidadores responsáveis pela criança e/ou adolescente atendidos no serviço Hot-line. Este atendimento telefônico presta orientações a pacientes e/ou seus cuidadores para tratar e resolver as crises de diabetes sem a necessidade de sair de casa.

Como critérios de inclusão utilizaram-se: ser cuidador de crianças e adolescentes diagnosticados com DM que utilizaram a ferramenta de telessaúde (Hot-Line) no período da pesquisa. Os critérios exclusão foram: ser participante sem acesso à internet para responder o questionário online e/ou sem número de telefone celular.

Após o atendimento, o participante foi informado que receberia uma mensagem via celular, contendo o convite com as explicações referentes ao projeto de pesquisa, o Termo de Consentimento Livre e Esclarecido e o link para direcioná-lo ao questionário online.

O questionário online, respondido pelo participante, determinava a resolutividade e satisfação quanto ao atendimento oferecido através deste serviço. $O$ instrumento consistia em três 
eixos principais: perfil do familiar e da criança ou adolescente, resolutividade da assistência e satisfação com a linha telefônica.

O primeiro eixo se referia ao perfil sociodemográfico do familiar e da criança ou adolescente e incluía fatores como idade, sexo, renda familiar, escolaridade e estado civil. $\mathrm{O}$ eixo 2 identificava o motivo da ligação através da Hot-Line e, conforme o motivo, o voluntário da pesquisa era conduzido às questões específicas do seu atendimento. Já no eixo 3 era abordada no questionário a avaliação quanto ao grau de satisfação com a assistência através deste serviço.

A análise dos dados foi realizada pelo software Statistical Package for Social Science (SPSS), versão 25.0 e as variáveis quantitativas foram avaliadas de forma descritiva, levando em consideração a frequência simples, média (para as variáveis com distribuição normal) e desvio- padrão. A estatística inferencial foi feita com os testes de qui-quadrado, teste t e correlação de Pearson. Foram consideradas diferenças estaticamente significativas quando o valor de $p$ foi inferior a 0,05.

$O$ estudo atendeu a todos os fundamentos éticos e científicos referentes às pesquisas que envolvem seres humanos, preconizadas pela Resolução no 466, do Conselho Nacional de Saúde, estabelecida em 12 de dezembro de $2012 .{ }^{14}$ O projeto foi submetido à análise e aprovado pelo Comitê de Ética e Pesquisa sob o número do parecer 2.828.424, em 29 de outubro de 2018.

\section{RESULTADOS}

Durante o período da coleta de dados, 316 registros de atendimentos da Hot-Line compuseram a amostra inicial do estudo. Contudo, destes, 90 cuidadores e/ou responsáveis de crianças e adolescentes responderam ao instrumento de pesquisa. Observa-se a média de idade dos cuidadores de $40,28+8,71$ anos. As características sociodemográficas dos participantes da pesquisa estão descritas na Tabela 1 a seguir.

Além das informações dos cuidadores, também foram coletados dados referentes às 90 crianças e adolescentes que receberam atendimento através da linha telefônica do serviço. O sexo prevalente entre as crianças foi o masculino $(58,9 \%)$, com idade média de $12,33+6,40$ anos, $97,8 \%$ dos pacientes com DM1. Em relação ao tempo de diagnóstico, pode-se observar que $28(31,4 \%)$ possuem 5 anos ou mais. No que tange à procedência, observou-se a predominância no contexto da Região Metropolitana de Porto Alegre (58,8\%). No entanto, pelo fato de o serviço ser referência no tratamento do Diabetes na região sul, houve chamadas telefônicas da fronteira e zona interiorana do estado, e do estado de Santa Catarina.

Os profissionais de saúde que mais atenderam às ligações da Hot-Line foram médicos(as) 69 (76,7\%), seguidos de enfermeiros(as) $20(22,2 \%)$ e $1(1,1 \%)$ nutricionista. Os motivos das ligações durante o atendimento encontram-se na Tabela 2.

Para o motivo de Ajuste de Dose, tiveram 41 cuidadores respondentes. Vale mencionar que a dose de insulina foi ajustada por orientações através de contato telefônico. Quarenta $(97,6 \%)$ afirmaram que o ajuste auxiliou no controle glicêmico. Em caso de hipoglicemia, 25 (61,0\%) diminuíram a dose, 5 (12,2\%) aumentaram a dose e para $11(26,8 \%)$ a dose permaneceu igual. Em caso de hiperglicemia, 5 (12,2\%) diminuíram a dose, 27 $(65,9 \%)$ aumentaram a dose e para $9(22,0 \%)$ a dose permaneceu igual. Após o ajuste, 28 (68,3\%) dos pacientes não necessitaram ligar novamente para o serviço de referência.

Quanto à Hiperglicemia Aguda, 6 responsáveis responderam ao questionário. Destes, apenas $1(16,7 \%)$ apresentou falta à escola, necessitando consultar outro serviço de saúde, sendo encaminhado para a emergência e, posteriormente, internação hospitalar. Quanto à Cetoacidose Diabética (CAD), 2 (33,3\%) dos pacientes fizeram tratamento para o quadro clínico.

Quanto à Hipoglicemia Aguda, 6 participantes responderam à pesquisa. Destes, 4 (66,7\%) apresentaram novo episódio de hipoglicemia, com variação numérica entre 1 e 10 novos episódios

Tabela 1. Perfil sociodemográfico dos cuidadores que responderam a pesquisa, Porto Alegre - RS, 2019

\begin{tabular}{|c|c|}
\hline Variáveis & $N(\%)$ \\
\hline \multicolumn{2}{|l|}{ CUIDADOR/RESPONSÁVEL } \\
\hline \multicolumn{2}{|l|}{ Parentesco } \\
\hline Mãe & $68(75,6)$ \\
\hline Pai & $12(13,3)$ \\
\hline Outros & $10(11,1)$ \\
\hline \multicolumn{2}{|l|}{ Sexo } \\
\hline Feminino & $76(84,4)$ \\
\hline Masculino & $14(15,6)$ \\
\hline \multicolumn{2}{|l|}{ Renda Familiar } \\
\hline$\geq 1 \mathrm{a}<2 \mathrm{SM}^{*}$ & $39(43,3)$ \\
\hline$\geq 2 \mathrm{a}<4 \mathrm{SM}$ & $13(14,4)$ \\
\hline$\geq 4 \mathrm{a}<6 \mathrm{SM}$ & $10(11,1)$ \\
\hline Não tem renda própria & $9(10,0)$ \\
\hline$<1 \mathrm{SM}$ & $8(8,9)$ \\
\hline$\geq 6 \mathrm{SM}$ & $7(7,8)$ \\
\hline Não sabe informar & $4(4,4)$ \\
\hline \multicolumn{2}{|l|}{ Escolaridade } \\
\hline Ensino médio completo & $39(43,3)$ \\
\hline Ensino superior completo & $14(15,6)$ \\
\hline Ensino médio incompleto & $11(12,2)$ \\
\hline Ensino fundamental completo & $10(11,1)$ \\
\hline Ensino superior incompleto & $9(10,0)$ \\
\hline Ensino fundamental incompleto & $7(7,8)$ \\
\hline \multicolumn{2}{|l|}{ Estado Civil } \\
\hline Casado/União estável & $56(62,2)$ \\
\hline Solteiro & $24(26,7)$ \\
\hline Outro & $10(11,1)$ \\
\hline
\end{tabular}

Fonte: Dados da pesquisa (2019). *SM = Salário Mínimo 
após a ligação. Dois (33,3\%) dos pacientes apresentaram perda de consciência e necessitaram consultar outro serviço de saúde, $1(16,7 \%)$ ao Centro Especializado e $1(16,7 \%)$ à emergência. Apenas $1(16,7 \%)$ apresentou falta escolar. Nenhum paciente necessitou de internação.

Referente ao motivo Dias de Doença, 2 pacientes responderam a pesquisa. Dois $(100,0 \%)$ consultaram outro serviço de saúde, e $1(50,0 \%)$ necessitou de internação hospitalar. Um paciente $(50,0 \%)$ precisou ligar novamente para o serviço de referência, buscando novas informações. Um paciente $(50,0 \%)$ apresentou hipoglicemia grave, e os 2 pacientes $(100,0 \%)$ apresentaram vômitos, dor abdominal e hálito de cetona na evolução do quadro.

$\mathrm{Na}$ tabela 3 está apresentado o grau de satisfação dos cuidadores de pacientes atendidos através da Hot-Line disponível

Tabela 2. Motivos da ligação durante atendimento aos cuidadores participantes da pesquisa, Porto Alegre - RS, 2019

\begin{tabular}{lc}
\hline \multicolumn{1}{c}{ Motivo da Ligação } & $\mathbf{N}(\%)$ \\
\hline Ajuste de Dose & $41(45,6)$ \\
\hline Orientações Gerais & $31(34,4)$ \\
\hline Hiperglicemia Aguda & $6(6,7)$ \\
\hline Hipoglicemia Aguda & $6(6,7)$ \\
\hline Resultados de Exames & $4(4,4)$ \\
\hline Dias de Doença & $2(2,2)$ \\
\hline
\end{tabular}

Fonte: Dados da pesquisa (2019).

Tabela 3. Grau de satisfação referente ao teleatendimento disponível no centro de referência em DM, Porto Alegre - RS, 2019

\begin{tabular}{ll}
\hline \multicolumn{1}{c}{ Grau de Satisfação } & N(\%) \\
\hline Orientação & $79(87,8)$ \\
\hline Boa/Ótima & $11(12,2)$ \\
\hline Regular/Insatisfatória & \\
\hline Utilidade & $80(88,9)$ \\
\hline Boa/Ótima & $10(11,1)$ \\
\hline Regular/Insatisfatória & \\
\hline Atendimento & $76(84,4)$ \\
\hline Boa/Ótima & $14(15,6)$ \\
\hline Regular/Insatisfatória & \\
\hline Profissional & $75(83,3)$ \\
\hline Boa/Ótima & $15(16,7)$ \\
\hline Regular/Insatisfatória & \\
\hline Tempo & $74(82,2)$ \\
\hline Boa/Ótima & $16(17,8)$ \\
\hline Regular/Insatisfatória &
\end{tabular}

Fonte: Dados da pesquisa (2019). no centro de referência. Pode-se observar que a maioria dos participantes da pesquisa considera boa/ótima a orientação recebida por contato telefônico.

O teste t entre a média da idade dos cuidadores e as avaliações do grau de satisfação não apresentou diferença estatística significativa $(p=0,914)$, mostrando um resultado geral, no qual a avaliação da satisfação foi independente da idade do cuidador. A avaliação foi independente do sexo $(p=0,202)$, quando comparado com o grau de satisfação, por meio do teste qui-quadrado. Quando comparado o parentesco com o grau de satisfação, não houve diferença estatística significativa $(p=0,303)$.

Existe diferença entre os níveis de escolaridade e a satisfação quanto à orientação $(p=0,032)$ e utilidade $(p=0,046)$, confirmadas pelo teste qui-quadrado, mostrando que os cuidadores que apresentam uma escolaridade mais completa demonstraram uma maior satisfação quanto à orientação recebida e à utilidade da Hot-Line.

As variáveis de caracterização como estado civil, raça, tempo de doença, frequência das ligações, profissional responsável pelo atendimento e motivo da ligação, quando comparadas com o grau de satisfação, não apresentaram diferença significativa $(p>0,05)$.

\section{DISCUSSÃO}

Neste estudo, pode-se perceber que o perfil dos cuidadores e/ou responsáveis que utilizam a linha telefônica foi em sua maioria do sexo feminino $76(84,4 \%)$, sendo o maior parentesco o materno 68 (75,6\%). Esse achado confirma que as mães são, na maioria das vezes, aquelas que assumem maiores responsabilidades no acompanhamento e participação no cuidado, principalmente ante a doença crônica na infância. ${ }^{15,16}$

Dentre os pacientes pediátricos com diabetes atendidos através da Hot-Line, o perfil sociodemográfico predominante foi de adolescentes do sexo masculino, com diagnóstico de DM1 e, tendo como tempo de diagnóstico da doença, entre 5 anos ou mais. Em razão da média de idade dos pacientes ser de 12,33 anos, ressalta-se a concordância com a mesma faixa etária em outro estudo. ${ }^{17}$ Nessa direção é importante que os pais e cuidadores saibam o tratamento das crianças e adolescentes, bem como ter consciência a respeito da sintomatologia e manejos no DM.

Em relação ao perfil sociodemográfico das crianças diagnosticadas com diabetes, existem divergências na literatura quanto ao sexo de maior prevalência. Alguns estudos corroboram com os achados deste estudo, apresentando o predomínio do sexo masculino. ${ }^{11,18}$ Contudo, há outro estudo no estado de Santa Catarina, que mostrou o predomínio do sexo feminino. ${ }^{19}$ E, um estudo retrospectivo desenvolvido em um ambulatório de endocrinologia pediátrica em São Paulo ressaltou que a frequência de ambos os sexos foi igual. ${ }^{20}$

O DM1 é o tipo mais prevalente na infância e adolescência, apresentando uma deficiência na secreção da insulina devido à destruição parcial ou total das células beta pancreáticas. ${ }^{1} \mathrm{O}$ presente estudo corrobora com os achados de Kharroubi ${ }^{1}$ e mostra concordância com as estatísticas, em 88 pacientes $(97,8 \%)$. 
Quanto ao tempo de doença, percebe-se que a maioria das crianças obteve o diagnóstico há mais de cinco anos. Esta característica foi oposta quando verificada em outro estudo, onde o tempo médio de diagnóstico foi menos de cinco anos. ${ }^{21}$ $\mathrm{Na}$ faixa pediátrica ocorrem picos de incidência do DM1. O primeiro é conhecido por ocorrer entre 4 e 6 anos de idade e está relacionado à exposição a agente infecciosos e o segundo dos 10 aos 14 anos, que apresenta relação com a puberdade e com a maior secreção do hormônio do crescimento, um hormônio que antagoniza a ação da insulina. ${ }^{19}$

Muitos são os cuidados necessários para o controle do diabetes em crianças e adolescentes, sendo a família um fator crucial no manejo e adesão ao tratamento. Na perspectiva da prática do cuidado à criança e ao adolescente, a questão que envolve os programas e propostas educativas para prevenção de doenças crônicas deve ser ampla, abrangendo meninos e meninas, desde a tenra idade à adolescência. Conviver com a doença e com a terapêutica dela derivada no caso do DM requer um enfoque diferenciado para os recursos cognitivos e emocionais que possam tornar as famílias aptas a desenvolver suas aptidões para cuidar das crianças.

A monitorização glicêmica é a melhor maneira de prevenção de complicações agudas, principalmente a hipoglicemia e de complicações crônicas ao longo do tempo. Para que isso ocorra, faz-se necessário incorporar conhecimentos a respeito dos sinais e sintomas de hipoglicemias e hiperglicemias nos pacientes pediátricos, além de agir rapidamente, sendo indispensável um contato acessível com a equipe de saúde. ${ }^{22}$

O motivo de ligação mais prevalente, do qual $45,6 \%$ dos cuidadores adquiriram assistência através do teleatendimento foi de ajustar a dose de insulina para adequar o controle glicêmico das crianças e adolescentes. Uma revisão sistemática discute que a possibilidade de disponibilizar um suporte adicional de telessaúde poderia qualificar habilidades de autocuidado no diabetes, minimizando complicações, melhorando desfechos, acarretando diminuição de idas às emergências e internações hospitalares. ${ }^{23}$

O uso da telemedicina vem-se tornando uma alternativa importante para a assistência em saúde, incluindo o cuidado para pacientes diabéticos, e apresenta potencial para superar barreiras impostas pela distância, tempo e eventuais despesas. ${ }^{24}$ A Sociedade Brasileira de Diabetes traz informações de que os serviços de saúde aprimoram o monitoramento e adesão ao tratamento dos pacientes ao adotar a utilização da tecnologia de informação, que consequentemente reduz as visitas às unidades de saúde. ${ }^{25}$

A literatura sugere que a telessaúde pode melhorar o manejo do diabetes, impactando a qualidade de vida e bemestar dos indivíduos. Em um estudo desenvolvido com crianças e adolescentes, observou-se que o grupo que utilizava a telemedicina apresentou melhoria tanto na glicose sanguínea quanto na hemoglobina glicada, quando comparado o grupo que não utilizava. ${ }^{26}$
Um importante aspecto da utilização das ferramentas de telessaúde é a sua capacidade de promover a educação em saúde aos pacientes pediátricos e suas famílias. O presente estudo possibilitou identificar que $34,4 \%$ das ligações foram específicas para orientações gerais. Outro aspecto fundamental para o controle da doença é a educação para o paciente diabético, retardando ou prevenindo o surgimento de complicações e promovendo a qualidade de vida. ${ }^{27}$

A equipe multiprofissional precisa continuamente procurar maneiras de compartilhar conhecimentos e informações para promover a qualificação no gerenciamento do diabetes nas habilidades necessárias para o autocuidado, encorajando o paciente a adotar novas práticas e mudanças de comportamento. ${ }^{21}$ A Sociedade Brasileira de Diabetes aponta que os principais objetivos da educação em diabetes são reduzir barreiras entre os pacientes com DM, suas famílias, comunidades e profissionais de saúde; capacitar para o autocuidado; melhorar resultados clínicos; prevenir complicações agudas e crônicas e proporcionar qualidade de vida. ${ }^{25}$

Destaca-se que a enfermagem apresenta um papel fundamental no processo de educação em saúde, por meio de uma relação de confiança, aproximando os profissionais e estimulando a participação ativa dos pacientes no plano de cuidados. Deve-se reconhecer a importância do nível de compreensão acerca da doença, visando garantir o entendimento da orientação recebida. Isso se intensifica ao considerarmos o contato telefônico como forma de disseminar conhecimentos. O acompanhamento por telefone permite o acesso às informações rapidamente, tornandose um instrumento facilitador da assistência. ${ }^{6}$

O U.S. Center for Medicare and Medicaid Services define o termo "Satisfação do Paciente" como a perspectiva e visão do cuidado recebido, que pode ser significativo para se comparar organizações de saúde. ${ }^{28} \mathrm{~A}$ satisfação do paciente é considerada um componente crucial da qualidade do cuidado que o paciente recebe e está diretamente conectada com maior adesão ao tratamento e desfecho clínico. ${ }^{29}$

No momento da ligação, $87,8 \%$ dos cuidadores afirmam que a orientação recebida foi ótima/boa. Oitenta $(88,9 \%)$ dos cuidadores consideram a utilidade da Hot-Line como ótima/boa. Dados similares foram encontrados em um estudo que buscou examinar o engajamento e satisfação com ligações de sistema de resposta interativa para manejo de doenças crônicas, onde $88 \%$ dos pacientes ficaram "muito satisfeitos" com este suporte e $100 \%$ reportaram que a orientação oferecida foi útil. ${ }^{30}$

A resolução 2.227/2018 do Conselho Federal de Medicina estabelece as normas éticas para essa modalidade de atendimento, sendo a resolução da Telemedicina mais atualizada no Brasil. Dentre o apresentado, destaca-se a teleconsulta, uma "consulta médica remota, mediada por tecnologias, com médico e paciente localizados em diferentes espaços geográficos", com prévio estabelecimento de uma relação presencial. ${ }^{31}$ No centro especializado, por se tratar de um serviço de saúde que atende pacientes com diabetes, há a periodicidade de consultas 
presenciais de 4 em 4 meses, garantindo, portanto, o aspecto legal da Hot-Line. Uma vez que a resolução estabelece que, em locais com atendimento especializado em "doenças crônicas, é recomendado consulta presencial em intervalos não superiores a 120 dias", para que seja disponibilizado a telemedicina como complemento do cuidado. ${ }^{31}$

\section{CONCLUSÕES E IMPLICAÇÕES PARA A PRÁTICA}

Constata-se que houve um alto índice de satisfação com o uso da linha telefônica, demonstrando seu papel efetivo no cotidiano das famílias, com orientações prestadas para a condição clínica no momento da ligação, auxiliando no autocuidado e controle adequado do DM. Recomenda-se, assim, ações de educação continuada junto à equipe de saúde, visando a qualificação do atendimento através da Hot-Line e potencializando a assistência prestada às famílias.

Além desse aspecto, ressalta-se que os resultados obtidos representam uma fração específica dentro da temática abordada, considerando que o tamanho amostral foi um fator limitante da pesquisa. Trabalhos futuros podem ser desenvolvidos, a fim de confirmar e ampliar os achados.

Por fim, acredita-se que os resultados desta pesquisa possam estimular reflexões sobre a utilização da telessaúde nos serviços, com o intuito de melhorar a qualidade do cuidado aos pacientes, contribuindo para promoção da saúde e prevenção de agravos nas crianças e adolescentes com diabetes.

\section{CONTRIBUIÇÕES DOS AUTORES}

Desenho do estudo. Coleta ou produção dos dados. Análise de dados e interpretação dos resultados. Redação e revisão crítica do manuscrito. Aprovação da versão final do artigo. Responsabilidade por todos os aspectos do conteúdo e a integridade do artigo publicado. Ana Carolina Schroder.

Análise de dados e interpretação dos resultados. Redação e revisão crítica do manuscrito. Aprovação da versão final do artigo. Responsabilidade por todos os aspectos do conteúdo e a integridade do artigo publicado. Ana Paula Vanz. Carolina Sturm Trindade

Desenho do estudo. Interpretação dos resultados. Redação e revisão crítica do manuscrito. Aprovação da versão final do artigo. Responsabilidade por todos os aspectos do conteúdo e a integridade do artigo publicado. César Geremia

Desenho do estudo. Análise de dados e interpretação dos resultados. Redação e revisão crítica do manuscrito. Aprovação da versão final do artigo. Responsabilidade por todos os aspectos do conteúdo e a integridade do artigo publicado. Simone Travi Canabarro

\section{EDITOR ASSOCIADO}

Gerson Luiz Marinho

\section{REFERÊNCIAS}

1. Kharroubi AT, Darwish HM. Diabetes mellitus: the epidemic of the century. World J Diabetes. 2015 jun;6(6):850-67. http://dx.doi.org/10.4239/wjd. v6.i6.850. PMid:26131326.

2. International Diabetes Federation. IDF Diabetes Atlas. 8th ed. Brussels, Belgium: IDF; 2017 [citado 2018 dez 13]. Disponível em: https://www. idf.org/e-library/epidemiology-research/diabetes-atlas/134-idf-diabetesatlas-8th-edition.html

3. Prytula Greco-Soares J, Dalbosco Dell'Aglio D. Relações entre qualidade de vida e diabetes mellitus tipo 1 na adolescência. Contextos Clín. 2016 jul;9(2):156-67. http://dx.doi.org/10.4013/ctc.2016.92.02.

4. Simões H, Serra F, Duarte S. Diabetes Tipo 2 na infância e adolescência - novos doentes, novos desafios. Revista Portuguesa de Diabetes. [Internet]. 2015; [citado 2018 dez 13];10(2):90-7. Disponível em: http:// www.revportdiabetes.com/wp-content/uploads/2017/11/RPD-Vol-10no-2-Junho-2015-Artigo-de-Revisão-págs-90-97.pdf

5. Barbosa D, Sousa F, Leite J. Scoring interventions in family relations regarding the care for the child with a chronic condition. Texto Contexto Enferm. 2015;24(1):87-95. http://dx.doi.org/10.1590/010407072015001820013.

6. Becker T, Teixeira C, Zanetti M. Nursing intervention in insulin administration: telephone follow-up. Acta Paul Enferm. 2012;25(spe 1):67-73. http:// dx.doi.org/10.1590/S0103-21002012000800011.

7. Tuckson RV, Edmunds M, Hodgkins M. Telehealth. N Engl J Med. 2017;377(16):1585-92. http://dx.doi.org/10.1056/NEJMsr1503323. PMid:29045204.

8. Kirsch SD, Wilson L, Harkins M, Albin D, Del Beccaro M. Feasibility of using a pediatric call center as part of a quality improvement effort to prevent hospital readmission. J Pediatr Nurs. 2015 mar;30(2):333-7. http://dx.doi.org/10.1016/j.pedn.2014.08.005. PMid:25193689.

9. Snoswell C, Smith A, Scuffham P, Whitty J. Economic evaluation strategies in telehealth: obtaining a more holistic valuation of telehealth interventions. J Telemed Telecare. 2017;23(9):792-6. http://dx.doi. org/10.1177/1357633X16671407. PMid:27789615.

10. International Diabetes Federation. IDF Diabetes Atlas [Internet]. 7th ed. Brussels, Belgium: IDF; 2015 [citado 2018 dez 13]. Disponível em: https://www.idf.org/e-library/epidemiology-research/diabetes-atlas/13diabetes-atlas-seventh-edition.html

11. Pennafort V, Silva A, Queiroz M. The perception of nurses regarding educational practices for children with diabetes in hospital care. Rev Gaúch Enferm. 2014;35(3):130-136. https://doi.org/10.1590/19831447.2014.03.43313.

12. Souza L, Figueiredo W, Machado M. As práticas de educação em diabetes vivenciadas no sus: uma discussão da literatura com ênfase na atenção primária à saúde. Rev APS. 2017;3(20):423-33. https://doi. org/10.34019/1809-8363.2017.v20.15801.

13. Gabarra L, Crepaldi M. A comunicação médico - paciente pediátrico família na perspectiva da criança. Psicol argum. 2011;29(65):209-18 http://doi.org/10.7213/rpa.v29i65.20335.

14. Resolução n. 466/2012 de 12 de dezembro de 2012 (BR). Trata de pesquisas em seres humanos e atualiza a resolução 196. Diário Oficial da União [periódico na internet], Brasília (DF), 12 dez 2012 [citado 2018 dez 13]. Disponível em: https://conselho.saude.gov.br/resolucoes/2012/ Reso466.pdf

15. Borsoi S, Scheidt I, Cordeiro G, Mascarenhas L. Análise da qualidade de vida em cuidadores de crianças e adolescentes com Diabetes tipo 1. Multitemas. 2018;23(55):25-39. http://dx.doi.org/10.20435/multi. v23i55.1743.

16. Piran P, Khademi Z, Tayari N, Mansouri N. Caregiving burden of children with chronic diseases. Electron Physician. 2017;9(9):5380-7. http:// dx.doi.org/10.19082/5380. PMid:29038725.

17. Lopes CLS, Pinheiro PP, Barberena LS, Eckert GU. Diabetic ketoacidosis in a pediatric intensive care unit. J Pediatr (Rio J). 2017;93(2):179-84. http://dx.doi.org/10.1016/j.jped.2016.05.008. PMid:27770618.

18. Silva A, Apolonio M, Alcantara C, Queiroz M. Características socioculturais e clínicas de adolescentes com Diabetes Mellitus tipo 1. Cogitare Enferm. 2016;21(4):1-8. http://dx.doi.org/10.5380/ce.v21i4.45699. 
19. Vargas D, Andrade B, Bork B. Perfil clínico e epidemiológico de crianças e adolescentes com Diabetes Mellitus 1 atendidos na atenção secundária em Blumenau-SC. ACM Arq Catarin Med. [Internet]. 2016; [citado 2018 dez 13];45(3):58-70. Disponível em: http://www.acm.org.br/acm/seer/ index.php/arquivos/article/view/111

20. Maruichi M, Takamune D, Noronha R, Schechtman H, Belhaus M, Kochi $\mathrm{C}$ et al. Características de crianças e adolescentes portadores de Diabetes Mellitus tipo 1 ao diagnóstico. Comparação entre dois períodos com dez anos de diferença em serviço universitário. Arq Méd Hosp Fac Ciênc Med Santa Casa São Paulo [Internet]. 2012; [citado 2018 dez 13];57(2):55-8. Disponível em: http://arquivosmedicos. fcmsantacasasp.edu.br/index.php/AMSCSP/article/view/281/293

21. Moreira T, Bandeira S, Lopes S, Carvalho S, Negreiros F, Neves C. Difficulties concerning Diabetes Mellitus Type 1 in children and adolescents. Rev Rene. 2016;17(5):651-8. http://dx.doi.org/10.15253/21756783.2016000500010.

22. Okido A, Almeida A, Vieira M, Neves E, Mello D, Lima R. As demandas de cuidado das crianças com Diabetes Mellitus tipo 1. Esc Anna Nery [online] 2017;21(2):e20170034. https://doi.org/10.5935/1414-8145.20170034.

23. Armstrong K, Moore MM. The impact of outpatient telehealth compared to standard care on emergency room visits and hospital admissions in pediatric diabetes patients: a systematic review protocol. JBI Database Syst Rev Implement Reports. 2018;16(1):63-70. http://dx.doi.org/10.11124/ JBISRIR-2016-003328. PMid:29324558.

24. Giani E, Laffel L. Opportunities and challenges of telemedicine: observations from the wild west in pediatric type 1 diabetes. Diabetes Technol Ther.2016;18(1):1-3. http://dx.doi.org/10.1089/dia.2015.0360. PMid:26756102.

25. Sociedade Brasileira de Diabetes. Diretrizes da Sociedade Brasileira de Diabetes 2017-2018. São Paulo: Editora Clannad; 2017 [citado
2018 dez 13]. Disponível em: https://www.diabetes.org.br/profissionais/ images/2017/diretrizes/diretrizes-sbd-2017-2018.pdf

26. Guljas R, Ahmed A, Chang K, Whitlock A. Impact of telemedicine in managing Type 1 diabetes among school-age children and adolescents: an integrative review. J Pediatr Nurs. 2014;29(3):198-204. http://dx.doi org/10.1016/j.pedn.2013.10.013. PMid:24269308.

27. Barbosa SA, Camboim FEF. Diabetes mellitus: cuidados de enfermagem para controle e prevenção de complicações. Temas em Saúde. [Internet]. 2016; [citado 2018 dez 13];16(3):404-417. [citado 2018 dez 13]. Disponível em: http://temasemsaude.com/wp-content/uploads/2016/09/16324.pdf

28. Kruse CS, Krowski N, Rodriguez B, Tran L, Vela J, Brooks M. Telehealth and patient satisfaction: a systematic review and narrative analysis. BM Open. 2017;7(8):e016242. http://dx.doi.org/10.1136/bmjopen-2017-016242. PMid:28775188.

29. Serrano $\mathrm{Cl}$, Shah $\mathrm{V}$, Abràmoff $\mathrm{MD}$. Use of expectation disconfirmation theory to test patient satisfaction with asynchronous telemedicine for diabetic retinopathy detection. Int J Telemed Appl. 2018;2018:1-14 http://dx.doi.org/10.1155/2018/7015272. PMid:30405712.

30. Piette JD, Marinec N, Gallegos-Cabriales EC, Gutierrez-Valverde JM, Rodriguez-Saldaña J, Mendoz-Alevares $M$ et al. Spanish-Speaking Patients' Engagement in Interactive Voice Response (IVR) chronic disease self-management support calls: analyses of data from three countries. J Telemed Telecare. 2013 fev;19(2):89-94. http://dx.doi. org/10.1177/1357633×13476234. PMid:23532005.

31. Resolução CFM n. 2.227/2018 (BR). Define e disciplina a telemedicina como forma de prestação de serviços médicos mediados por tecnologias. Diário Oficial da União [periódico na internet], Brasília (DF), 6 fev 2019 : Seção 1:58 [citado 2018 dez 13]. Disponível em: https://portal.cfm.org. br/images/PDF/resolucao222718.pdf 